\title{
CLASSIFICATIONS OF 2-COMPLEXES WHOSE FINITE FUNDAMENTAL GROUP IS THAT OF A 3-MANIFOLD
}

\author{
by F. RUDOLF BEYL, M. PAUL LATIOLAIS and NANCY WALLER
}

(Received 9th January 1995)

\begin{abstract}
We consider spines of spherical space forms; i.e., spines of closed oriented 3-manifolds whose universal cover is the 3-sphere. We give sufficient conditions for such spines to be homotopy or simple homotopy equivalent to 2-complexes with the same fundamental group $G$ and minimal Euler characteristic 1. If the group ring $\mathbb{Z} G$ satisfies stably-free cancellation, then any such 2-complex is homotopy equivalent to a spine of a $3-$ manifold. If $K_{1}(\mathbb{Z} G)$ is represented by units and $K$ is homotopy equivalent to a spine $X$, then $K$ and $X$ are simple homotopy equivalent. We exhibit several infinite families of non-abelian groups $G$ for which these conditions apply.
\end{abstract}

1991 Mathematics subject classification: Primary 57M20; Secondary 57Q10, 57M60, 19B28, 19 A31, 19 A13.

\section{Introduction}

Considerable interest has developed recently in the algebraic topology of 2complexes, particularly that of spines of 3-manifolds. Historically, 2-complexes were thought to be a possible tool in the study of 3-manifolds. The obvious connections did not work out. For example, there are many distinct lens spaces with the same spine [6]. Recently developed connections and tools continue to give us new insights into the topology of manifolds and the study of finite groups $[8,14,23]$.

Our interests are the connection between the Generalized Andrews-Curtis Conjecture [10] and Thurston's Geometrization Conjecture [24] (for finite fundamental groups). The Andrews-Curtis Conjecture, stated topologically, says that every finite 2-complex with trivial fundamental group 3-deforms to a wedge of spheres [10]. For Euler characteristic 1, this would mean such a 2-complex would 3-deform to a point. A counter-example to Andrews-Curtis which is also a spine of a 3-manifold would give a counter-example to the Poincaré Conjecture [10].

Metzler [17] gave the first examples of 2-complexes with the same (non-trivial) fundamental group and same Euler characteristic which are not homotopy equivalent. These examples gave rise to two questions [3]:

(1) Does homotopy equivalence imply simple homotopy equivalence for 2complexes?

For all finite abelian fundamental groups, homotopy equivalence does imply simple 
homotopy equivalence for 2-complexes [12]. For finite non-abelian fundamental groups, partial results are known $[7,13]$. In general, there exist homotopy equivalent 2-complexes (with infinite fundamental group) which are not simple homotopy equivalent $[15,18]$.

(2) Does simple homotopy equivalence of finite 2-complexes imply equivalence via 3-deformations?

This question is called the Generalized Andrews-Curtis Conjecture. A 3-deformation is a sequence of elementary expansions and collapses, where all of the intermediate spaces have dimension 3 or less. A counter-example pair with finite fundamental group, both of which are spines of a 3-manifold, would also give a counter-example to Thurston's Geometrization Conjecture. This can be seen in the following way:

Thurston's Geometrization Conjecture, if true, would imply in particular that all closed 3-manifolds with finite fundamental group would arise as quotients of orthogonal actions on $S^{3}$. Since an orthogonal action on $S^{3}$ gives rise to a Seifert fibration, Thurston's Conjecture would imply that all closed 3-manifolds with finite fundamental group are Seifert fibered. Now, using Threlfall and Seifert [29], for any given non-cyclic finite fundamental group there is exactly one closed Seifert fibered 3-manifold. Consequently, Thurston's Conjecture would imply that there is exactly one closed 3-manifold corresponding to each non-cyclic finite fundamental group. In particular, every 3-manifold spine with a given non-cyclic finite fundamental group would 3-deform to every other one, as they would be spines of the same 3-manifold.

In this paper, we take the first steps in the study of the connection between the Generalized Andrews-Curtis Conjecture and Thurston's Geometrization Conjecture. That is, we study the homotopy classifications of 2-complexes whose fundamental group acts orthogonally* on $S^{3}$. In particular, we consider only finite groups in this paper.

Finite groups that admit a fixed-point-free orthogonal* action on $S^{3}$ are given by the following four classes $[19,28]$ :

(I) The binary polyhedral groups:

the binary dihedral groups $\left.Q_{4 n}=\langle x, y| x^{2}=(x y)^{2}=y^{n}\right), n \geq 2$;

the binary tetrahedral group $T_{24}=\left\langle x, y \mid x^{2}=(x y)^{3}=y^{3}, x^{4}=1\right\rangle$;

the binary octahedral group $O_{48}=\left\langle x, y \mid x^{2}=(x y)^{3}=y^{4}, x^{4}=1\right\rangle$;

the binary icosahedral group $I_{120}=\left\langle x, y \mid x^{2}=(x y)^{3}=y^{5}, x^{4}=1\right\rangle$.

(II) The groups

$$
D\left(2^{k}, 2 l+1\right)=\left\langle x, y \mid x^{2^{k}}=1, y^{2 l+1}=1, x y x^{-1}=y^{-1}\right\rangle, \quad k \geq 3, l \geq 1 .
$$

* Added in proof (August 1996): Ian Hambleton and Ronnie Lee just announced their solution of the Spherical Space Form Problem: The list of finite groups acting on a closed connected 3-manifold consists only of those groups that can act orthogonally on the 3-sphere. Thus the groups considered in this paper are all finite 3-manifold groups. 
(III) The groups

$$
T\left(8,3^{k}\right)=\left\langle x, y, z \mid x^{2}=(x y)^{2}=y^{2}, z^{3^{k}}=1, z x z^{-1}=y, z y z^{-1}=x y\right\rangle, \quad k \geq 2 .
$$

(IV) Cyclic groups $C_{m}$ and direct products $C_{m} \times G$, where $G$ is any group in class (I), (II), or (III), with order relatively prime to $m$.

We thank William A. Bogley, Martin Lustig, Robert Oliver, Allan Sieradski and the referee for their assistance.

\section{Homotopy types of some 2-complexes}

In this section we discuss the homotopy type of minimal $(G, 2)$-complexes, where $G$ is finite and admits a free orthogonal action on $S^{3}$. A minimal $(G, 2)$-complex is a 2-dimensional CW-complex $K$ whose Euler characteristic is minimal with respect to the fundamental group $\pi_{1}(K) \cong G$. The following hypothesis will hold throughout the next two sections:

Hypothesis 1.1. Let $M$ be a closed connected orientable 3-manifold whose fundamental group $G$ is finite.

Suppose $M$ satisfies (1.1). We may assume that $M$ is a $P L C W$-complex (see [10, Chapter 1]). We may view $M$ as consisting of a 2-complex $X$, the spine, and a single attached 3-cell which intersects each open 2-cell of $X$ exactly twice. This can be seen in the following way. The $P L C W$ structure may be suitably subdivided to be simplical. Consider a maximal tree in the dual complex. Adjacent vertices in this tree correspond to 3-cells in $M$ which share a boundary 2-cell. We may remove this common 2-cell and replace the adjoining 3-cells by a single 3-cell, attached by the obvious combinatorial characteristic map. Starting from a terminal branch of the tree, we may use this procedure to inductively define a single 3-cell that contains the interiors of all the original 3-cells. Since each 2-cell lies in the boundary of exactly two of the original 3-cells, any open 2-cell in the boundary of this new 3-cell appears exactly twice. An orientation on the 3-cell induces opposite orientations on the two copies of this 2-cell.

A spine of a 3-manifold satisfying (1.1) is minimal, as it has Euler characteristic 1. Such a 2-complex may be assumed to have a single 0 -cell by collapsing a maximal tree in the 1-skeleton. The group presentation associated to this collapsed 2-complex is balanced.

Proposition 1.2. Let $X$ be a $(G, 2)$-complex which is a spine of a 3-manifold $M$ satisfying (1.1). Then $\pi_{2}(X) \cong \mathbb{Z G / N G}$, where $N$ is the sum of all group elements and $N G$ is the ideal generated by $N$. In particular, assuming $X$ has a single 0 -cell, $\pi_{2}(X)$ is generated by an element of the form

$$
\left(g_{1}-1\right) S_{1}+\left(g_{2}-1\right) S_{2}+\ldots+\left(g_{n}-1\right) S_{n}
$$

where the $S_{i}$ are 2-cells representing relators and the $g_{i}$ generate the group. 
Proof. We may assume that $M$ has the combinatorial $C W$-structure given above, with a single 3-cell $c^{3}$ and a single 0 -cell $c^{0}$. Remove an open ball $B$ from the interior of the 3-cell. The spine $X$ is a deformation retract of $M-B$, where $\partial B$ is radially deformed to the boundary of the single 3-cell. Now consider a preferred lift $\tilde{B}$ of $B$ in the universal cover $\tilde{M}$. The 3 -manifold $\tilde{M}-\{g \cdot \tilde{B} \mid g \in G\}$ is simply connected by a Seifert-Van Kampen argument and is the universal cover of $M-B$. By Hurewicz, $\pi_{2}(X) \cong H_{2}(M \widetilde{-B})$. The 2-cycles consist of the free abelian group generated by $\{g \cdot \partial \tilde{B} \mid g \in G\}$, while $\partial(\widetilde{M-B})=\Sigma g \cdot \partial \tilde{B}$ generates the 2 -boundaries. Thus $\pi_{2}(X) \cong \mathbb{Z} G / N G$, generated by $\partial \tilde{B}$.

The deformation retraction $r: M-B \searrow X$ identifies the image $r(\partial B)$ with the boundary of the 3-cell $c^{3}$. This lifts to a deformation retraction $\tilde{r}$ of the universal cover $M \simeq B$. Choose a lift $\tilde{c}^{3}$ of $c^{3}$ and a lift $\tilde{B}$ of $B$ with $\tilde{B} \subseteq \tilde{c}^{3}$. Let $\tilde{c}^{0} \subseteq \tilde{r}(\partial \tilde{B})$ be a lift of $c^{0}$. Since $r(\partial B)$ meets each open 2-cell of $X$ exactly twice, once in each orientation, and meets every 2 -cell at the single vertex $c^{0}$, we may represent $\tilde{r}(\partial \tilde{B})$ as $\Sigma\left(1-g_{i}\right) \cdot S_{i}$, where the $S_{i}$ are 2-cells and $g_{i} \in G$.

Now consider the 1 -skeleton of the dual complex, $\tilde{M}_{D}^{1}$, and let $s t \tilde{c}^{3}$ denote the star of the vertex corresponding to $\tilde{c}^{3}$ in $\tilde{M}_{D}^{1}$. Since $G \cdot s t \tilde{c}^{3}=\tilde{M}_{D}^{1}$ and $\tilde{M}_{D}^{1}$ is connected, the set $\left\{g \mid g \cdot s t \tilde{c}^{3} \cap s t \tilde{c}^{3} \neq \emptyset\right\}=\left\{g_{i}, g_{i}^{-1} \mid i=1,2, \ldots, n\right\}$ generates $G([25$, p. 30]).

Whenever we refer to a $(G, 2)$-complex $X$ that is a spine of a 3 -manifold, we can (and will) assume that $X$ has a single 0 -cell and $\pi_{2}(X)$ has a generator of the form given in the preceding proposition.

Now let $K$ be any minimal $(G, 2)$-complex. By shrinking a maximal tree in the 1 -skeleton, we may assume that $K$ is a complex associated with some presentation of $G$ given by $P_{K}=\langle\mathbf{y} \mid \mathbf{r}\rangle$. Let $P_{X}=\langle\mathbf{z} \mid \mathbf{s}\rangle$ be the presentation associated with the spine $X$. Given an isomorphism $\alpha: \pi_{1}(X) \rightarrow \pi_{1}(K)$, the images of the generators $z_{i}$ can be expressed as words in the generators $y_{j}$ of $\pi_{1}(K), \alpha\left(z_{i}\right)=w_{i}(y)$. We form the expanded presentation for $\pi_{1}(K)$ :

$$
\left.\langle\mathbf{y}, \mathbf{z}| \mathbf{r}, z_{i}^{-1} w_{i}(\mathbf{y}) \text { for } z_{i} \in \mathbf{z}\right\rangle .
$$

The complex associated with this presentation is obtained from $K$ by elementary cellular expansions and therefore is simple homotopy equivalent to $K$. Similarly, we can expand $X$ to a presentation complex on generators $\mathrm{z} \cup \mathrm{y}$ by using $\alpha^{-1}$.

Thus from now on we assume that $X$ and $K$ have identical 1-skeletons when considering the problem of their homotopy equivalence-or even simple homotopy equivalence. However, one must keep in mind that the identification of the 1-skeletons comes from the map $\alpha$. When we identify 1-skeletons, we may not assume that an equivalence will respect this identification. The equivalences we construct will, in general, respect a given identification of 1-skeletons. Note also that the second homotopy group of the expanded spine is still singly generated by the element (1.3).

Consider the two partial free $\mathbb{Z} G$-resolutions of $\mathbb{Z}$ given by the chain complexes of the universal covers of $X$ and $K$, respectively. Given that the 1-skeletons are identified, Schanuel's Lemma yields 


$$
\pi_{2}(K) \oplus C_{2}(\tilde{X}) \cong \pi_{2}(X) \oplus C_{2}(\tilde{K}) .
$$

Since the Euler characteristics of $X$ and $K$ are equal, the ranks of the free $\mathbb{Z} G$ summands added to each side have the same value, say $k$. By Proposition 1.2

$$
\pi_{2}(K) \oplus[\mathbb{Z} G]^{k} \cong \mathbb{Z} G / N G \oplus[\mathbb{Z} G]^{k}
$$

Definition 1.5. The group ring $\mathbb{Z} G$ has stably-free cancellation if every stably-free module is free; that is, whenever a $\mathbb{Z} G$-projective $P$ satisfies $P \oplus \mathbb{Z} G \cong[\mathbb{Z} G]^{k} \oplus \mathbb{Z} G$ for finite $k$, we have $P \cong[\mathbb{Z} G]^{k}$.

A stronger version of the following proposition is due to Dyer ([5, Prop. 5.4]). For completeness, we supply a proof for the version needed here.

Proposition 1.6. Let $\mathbb{Z} G$ have stably-free cancellation. If $M$ is a $\mathbb{Z} G$-module such that $M \oplus[\mathbb{Z} G]^{k} \cong \mathbb{Z} G / N G \oplus[\mathbb{Z} G]^{k}$ for finite $k$, then $M \cong \mathbb{Z} G / N G$.

Proof. Consider the exact sequence

$$
0 \longrightarrow N G \stackrel{i}{\longrightarrow} \mathbb{Z} G \stackrel{\curvearrowright}{\longrightarrow} \mathbb{Z} G / N G \longrightarrow 0
$$

Given some isomorphism $\beta: \mathbb{Z} G / N G \oplus[\mathbb{Z} G]^{k} \cong M \oplus[\mathbb{Z} G]^{k}$, we have the exact sequence

$$
0 \longrightarrow N G \stackrel{[i, 0]}{\longrightarrow} \mathbb{Z} G \oplus[\mathbb{Z} G]^{k} \stackrel{\beta[2 \oplus i d]}{\longrightarrow} M \oplus[\mathbb{Z} G]^{k} \longrightarrow 0 .
$$

Let $p_{2}: M \oplus[\mathbb{Z} G]^{k} \rightarrow[\mathbb{Z} G]^{k}$ be the projection onto the second factor. Since $[\mathbb{Z G}]^{k}$ is projective, there exists a map $h:[\mathbb{Z G}]^{k} \rightarrow \mathbb{Z} G \oplus[\mathbb{Z} G]^{k}$ such that $p_{2} \beta[\alpha \oplus i d] h=i d_{[\mathbf{Z G}]^{k}}$.

Let $P=\operatorname{ker}\left\{p_{2} \beta[\alpha \oplus i d]\right\}$. Then $\operatorname{im}[i, 0] \subseteq P$, and $\mathbb{Z} G \oplus[\mathbb{Z} G]^{k}$ is the internal direct sum of $P$ and $i m h$. Since $i m h \cong[\mathbb{Z} G]^{k}$, our hypothesis implies $P \cong \mathbb{Z} G$. These identifications give a change of basis in $\mathbb{Z} G \oplus[\mathbb{Z} G]^{k}$, under which (1.7) takes the form

$$
0 \longrightarrow N G \stackrel{[j, 0]}{\longrightarrow} \mathbb{Z} G \oplus[\mathbb{Z} G]^{k} \stackrel{\left[\begin{array}{ll}
\gamma & 0 \\
\delta & I
\end{array}\right]}{\longrightarrow} M \oplus[\mathbb{Z} G]^{k} \longrightarrow 0
$$

where the given matrices act on the right. Since the inverse image of $M \oplus \mathbf{O}$ in (1.8) is contained in $\mathbb{Z} G \oplus 0, \gamma$ is surjective. By restricting our attention to the first factor, we extract the exact sequence

$$
0 \longrightarrow N G \stackrel{j}{\longrightarrow} \mathbb{Z} G \stackrel{?}{\longrightarrow} M \longrightarrow 0 .
$$

The result follows upon showing that $i m j$ is $N G$ itself, not just some isomorphic copy. 
Since $N G$ is largest submodule of $\mathbb{Z} G$ with trivial $G$-action and $j$ is $G$-linear, we have im $j \subseteq N G$. Now observe that $M$ is annihilated by $N$, since $M \oplus[\mathbb{Z} G]^{k} \cong \mathbb{Z} G / N G \oplus[\mathbb{Z} G]^{k}$ implies $N M \oplus[Z]^{k} \cong[Z]^{k}$ as abelian groups. Thus $N G \subseteq \operatorname{ker} \gamma=i m j$.

Proposition 1.9. Let $K$ be a minimal $(G, 2)$-complex where $G$ is the fundamental group of a 3-manifold $M$ satisfying (1.1) with spine $X$. Assume that $\mathbb{Z} G$ satisfies stably-free cancellation, or that $\pi_{2}(K)$ is singly generated. Then $\pi_{2}(K) \cong \mathbb{Z} G / N G \cong \pi_{2}(X)$.

Proof. If $\mathbb{Z} G$ satisfies stably-free cancellation, then the conclusion follows from (1.4) and Proposition 1.6. On the other hand, if $\pi_{2}(K)$ is singly generated, there is an epimorphism $\alpha: \mathbb{Z} G \rightarrow \pi_{2}(K)$. By (1.4), we have $\pi_{2}(K) \oplus[\mathbb{Z} G]^{k} \cong \mathbb{Z} G / N G \oplus[\mathbb{Z} G]^{k}$, hence $N \cdot \pi_{2}(K) \oplus \mathbb{Z}^{k} \cong \mathbb{Z}^{k}$ as abelian groups, and $N$ annihilates $\pi_{2}(K)$. Thus $N \in$ ker $\alpha$ and $\alpha$ factors through an epimorphism $\beta: \mathbb{Z} G / N G \rightarrow \pi_{2}(K)$. Now $\operatorname{rank}(\mathbb{Z} G / N G)=|G|-1$ as a free abelian group. Since $K$ has minimal Euler characteristic 1, we have

$$
|G|=\chi(\tilde{K})=\operatorname{rank}\left[H_{0}(\tilde{K})\right]-\operatorname{rank}\left[H_{1}(\tilde{K})\right]+\operatorname{rank}\left[H_{2}(\tilde{K})\right]=1-0+\operatorname{rank}\left[\pi_{2}(K)\right] .
$$

Thus $\beta$ is an isomorphism, since $\mathbb{Z} G / N G$ and $\pi_{2}(K)$ have the same free abelian rank.

Theorem 1.10. Let $K$ be a minimal $(G, 2)$-complex where $G$ is the finite fundamental group of a closed orientable 3-manifold $M$. Suppose that $\mathbb{Z} G$ has stably-free cancellation. Then $K$ is homotopy equivalent to a spine of $M$.

Proof. We will show how to construct a cellular map from a spine $X$ to $K$ which induces isomorphisms on both the first and second homotopy groups. By Whitehead's theorem [30], this will give a homotopy equivalence. The preceding discussion shows that we may assume that $K$ and $X$ have identical 1 -skeletons. Consider any cellular map $f: X \rightarrow K$ extending the identity on the 1 -skeleton, and the induced maps on the universal cover

$$
\begin{aligned}
& 0 \rightarrow \pi_{2}(X) \stackrel{i}{\rightarrow} C_{2}(\tilde{X}) \rightarrow C_{1}(\tilde{X}) \rightarrow C_{0}(\tilde{X}) \rightarrow \mathbb{Z} \rightarrow 0 \\
& \tilde{f}_{2}^{\#} \downarrow \quad \tilde{f}_{2} \downarrow \quad \tilde{f}_{1}\left\|\quad \tilde{f}_{0}\right\| \quad \| \\
& 0 \rightarrow \pi_{2}(K) \stackrel{j}{\rightarrow} C_{2}(\tilde{K}) \rightarrow C_{1}(\tilde{K}) \rightarrow C_{0}(\tilde{K}) \rightarrow \mathbb{Z} \rightarrow 0
\end{aligned}
$$

We will use a Puppe modification ([12, Lemma 1.4, p. 656]) to change the action of $f$ on 2-cells (but leaving the identification of the 1-skeletons unchanged), so that the new map is a homotopy equivalence. Such modifications result in replacing $\tilde{f}_{2}$ by $\tilde{f}_{2}+j \circ \gamma$ and $\tilde{f}_{2}^{\#}$ by $\tilde{f}_{2}^{\#}+\gamma \circ i$, where $\gamma: C_{2}(\tilde{X}) \rightarrow \pi_{2}(K)$ is arbitrary. For this construction, recall that $\pi_{2}(X) \cong \mathbb{Z G} / N G \cong \pi_{2}(K)$ by Proposition 1.9, and let $\sigma, \tau$ generate $\pi_{2}(X), \pi_{2}(K)$, respectively. Let $S_{1}, S_{2}, \ldots, S_{n}$ be the usual basis for $C_{2}(\tilde{X})$. Then $\tilde{f}_{2}^{\#}(\sigma)=\eta \cdot \tau$, where $\eta \in \mathbb{Z} G$ is determined up to a multiple of $N$. Given $A_{1}, A_{2}, \ldots, A_{n} \in \mathbb{Z} G$, we may replace $f$ by a map $h$ with 


$$
\tilde{h}_{2}\left(S_{i}\right)=\tilde{f}_{2}\left(S_{i}\right)+A_{i} \cdot \tau \quad i=1,2, \ldots, n .
$$

Applying $\tilde{h}_{2}^{\#}$ to the generator $\sigma=\left(g_{1}-1\right) S_{1}+\left(g_{2}-1\right) S_{2}+\ldots+\left(g_{n}-1\right) S_{n}$ gives

$$
\tilde{h}_{2}^{\#}(\sigma)=\left[\eta+\left(g_{1}-1\right) A_{1}+\left(g_{2}-1\right) A_{2}+\ldots+\left(g_{n}-1\right) A_{n}\right] \cdot \tau .
$$

Since the $A_{i}$ are arbitrary and the $g_{i}$ generate $G$, we may use this process to replace $\eta$ by any element of $\mathbb{Z} G$ with the same augmentation mod $|G|$.

The map $\tilde{f}_{2}^{\#}$, together with all Puppe modifications, represents a $k$-invariant class; that is, an element of

$$
H^{3}\left(G ; \pi_{2}(K)\right) \cong \operatorname{Hom}\left(\pi_{2}(X), \pi_{2}(K)\right) / i^{*} \operatorname{Hom}\left(C_{2}(\tilde{X}), \pi_{2}(K)\right) \cong \mathbb{Z} /|G| \mathbb{Z}
$$

[2]. Here we specifically identify $\operatorname{Hom}\left(\pi_{2}(X), \pi_{2}(K)\right) / i^{*} \operatorname{Hom}\left(C_{2}(\tilde{X}), \pi_{2}(K)\right)$ with $\mathbb{Z} /|G|$ by choosing $[k] \in \mathbb{Z} /|G|$ to represent the class of the map given by multiplication by $k \bmod |G|: \sigma \rightarrow k \cdot \tau$. Thus $\left[\tilde{f}_{2}^{\#}\right]=[k]$ where $k=\epsilon(\eta) \bmod |G|$. Dyer $([5$, Theorem 3.5]) has shown that if a $k$-invariant can be realized by a map between cellular chain complexes then the Swan projective $\langle k, N\rangle$ is stably-free with $\langle k, N\rangle \oplus \mathbb{Z} G \cong \mathbb{Z} G \oplus \mathbb{Z} G$. Our cancellation hypothesis then ensures that $\langle k, N\rangle \cong \mathbb{Z} G$. Thus there exists a unit in $\mathbb{Z} G / N G$ with augmentation $k \bmod |G|([26$, Lemma 6.3]). By an appropriate choice of $A_{1}, A_{2}, \ldots, A_{n}$, we may now use a Puppe modification to replace the map $f$ with a map $h$ such that the $\eta$ defined by $\tilde{f}_{2}^{\#}$ is replaced by this unit. Then $\tilde{h}_{2}^{\#}: \pi_{2}(X) \cong \pi_{2}(K)$. Since $h$ maintains the identification of the l-skeletons, we have constructed a homotopy equivalence.

If it is already known that $\pi_{2}(K) \cong \mathbb{Z} G / N G$, inspection of the above proof of Theorem 1.10 shows that the hypothesis of stably-free cancellation may be weakened.

Definition 1.11. A group $\mathbb{Z} G$ satisfies weak cancellation if every stably-free Swan projective $\langle k, N\rangle$ is free.

Corollary 1.12. Let $K$ be a minimal $(G, 2)$-complex where $G$ is the finite fundamental group of a closed orientable 3-manifold $M$. Suppose $\pi_{2}(K)$ is singly generated and that $\mathbb{Z} G$ satisfies weak cancellation. Then $K$ is homotopy equivalent to a spine of $M$.

Proof. This follows from Proposition 1.9 and the proof of Theorem 1.10.

\section{Simple homotopy types of some 2-complexes}

We have seen that there are many examples where minimal $(G, 2)$-complexes, with $G$ the fundamental group of a closed 3-manifold $M$, are all homotopy equivalent to a spine of $M$. It is natural to ask whether they are also simple homotopy equivalent. 
Definition 2.1. An element $x$ in $K_{1}(\mathbb{Z} G)$ is represented by a unit of $\mathbb{Z} G$ if there exists a $\mu$ in the unit group $\mathbb{Z} G^{*}$ such that $x=[\mu]$ in $K_{1}(\mathbb{Z} G)$. We say the $K_{1}(\mathbb{Z} G)$ is represented by units if each of its elements is.

Lemma 2.2 Let $R$ be a ring with unity and $M \in M_{n}(R)$ be a square matrix of the form

$$
M=I+c \cdot r^{T}
$$

where $I$ is the $n \times n$ identity matrix and $c, r$ are $n$-dimensional column vectors.

Then

$$
\left[\begin{array}{cc}
M & 0 \\
0 & 1
\end{array}\right] \sim\left[\begin{array}{cc}
1+r^{T} \cdot c & 0 \\
0 & I
\end{array}\right]
$$

is an equivalence under elementary row and column operations, in the sense of Cohen [3]. In particular, $M$ is invertible if and only if $1+r^{T} \cdot c$ is a unit of $R$, in which case $M$ is represented by this unit in $K_{1}(R)$.

Proof.

$$
\begin{gathered}
{\left[\begin{array}{cc}
I+c \cdot r^{T} & 0 \\
0 & 1
\end{array}\right] \sim\left[\begin{array}{cc}
I+c \cdot r^{r} & -c \\
0 & 1
\end{array}\right] \sim\left[\begin{array}{cc}
I & -c \\
r^{T} & 1
\end{array}\right]} \\
\sim\left[\begin{array}{cc}
I & 0 \\
r^{T} & 1+r^{T} \cdot c
\end{array}\right] \sim\left[\begin{array}{cc}
I & 0 \\
0 & 1+r^{T} \cdot c
\end{array}\right] \sim\left[\begin{array}{cc}
1+r^{T} \cdot c & 0 \\
0 & I
\end{array}\right],
\end{gathered}
$$

where each step follows from ([3, p. 38]).

Lemma 2.3. Let $X$ be a spine of a closed orientable 3-manifold $M$ with fundamental group $G$. Every unit $\mu \in \mathbb{Z} G$ can be realized as the Whitehead torsion of some self equivalence $h: X \cong X$.

Proof. Every unit $\mu$ of $\mathbb{Z} G$ has augmentation \pm 1 . Since Whitehead torsion lives in $K_{1}(\mathbb{Z} G) / \pm G$, we may assume that $\mu$ has augmentation +1 . Recall that $\pi_{2}(X)$ has a generator of the form $\sigma=\Sigma\left(1-g_{i}\right) \cdot S_{i}$ where the $g_{i}$ generate $G$, and the $S_{i}$ correspond to 2-cells in a complex associated with some presentation of $G$. Since $\epsilon(\mu)=1$, we have $\mu=1+\Sigma\left(1-g_{i}\right) \cdot \alpha_{i}$ for some choice of $\alpha_{i} \in \mathbb{Z} G$. Define a cellular map $h: X \rightarrow X$ which is the identity on the l-skeleton, and which induces a map $\tilde{h}_{2}: C_{2}(\tilde{X}) \rightarrow C_{2}(\tilde{X})$ given by

$$
\tilde{h}_{2}\left(S_{i}\right)=S_{i}+\alpha_{i} \cdot \sigma
$$

Note that a Puppe modification of the identity map can be used to obtain the map $h$. 
One may verify that $h$ induces multiplication by $\mu$ on $\pi_{2}(X)$. Lemma 2.2 shows that the matrix representing $\tilde{h}$ is equivalent to $[\mu]$ in $K_{1}(\mathbb{Z} G)$.

Theorem 2.4. Suppose that $K_{1}(\mathbb{Z} G)$ is represented by units. Let $K$ be a $(G, 2)$-complex which is homotopy equivalent to a spine $X$ of a closed, orientable 3-manifold. Then $K$ and $X$ are simple homotopy equivalent.

Proof. We may assume that $K$ and $X$ have identical 1 -skeletons and that the homotopy equivalence $f: X \rightarrow K$ preserves this identification. In this situation $\tilde{f}_{2}: C_{2}(\tilde{X}) \rightarrow C_{2}(\tilde{K})$ is an isomorphism. The Whitehead torsion of $f$ is given by the class of the matrix $\tilde{M}_{2}$, which represents the induced map $\tilde{f}_{2}$ with respect to the standard bases [3]. By hypothesis, $\tilde{M}_{2}$ is equivalent to a unit $[\mu]$ in $K_{1}(\mathbb{Z} G)$. By Lemma $2.3,\left[\mu^{-1}\right]$ is represented by the torsion of a self equivalence $h: X \rightarrow X$. The composition $f h$ is a simple homotopy equivalence.

\section{Examples}

In this section we give examples of groups $G$ that satisfy stably-free cancellation, weak cancellation, or for which $K_{1}(\mathbb{Z} G)$ is represented by units. We also point out cases where these conditions fail. This is not intended to be an exhaustive list. All of our examples act orthogonally on $S^{3}$. Most arguments of this section were suggested to us by Robert Oliver, in particular the proof of Theorem 3.5 is due to him.

\section{Cancellation Properties}

The cancellation properties connected with binary polyhedral groups were studied by Swan [27]. He defined cancellation to hold for a ring $R$ provided that $R \oplus P \cong R \oplus Q$ implies $P \cong Q$ for finitely generated projectives $P$ and $Q$. Cancellation for $\mathbb{Z} G$ implies stably-free cancellation, and either implies weak cancellation. The following are direct consequences of his results:

(1) ([27, Theorem I, p. 66]) Let $G$ be a binary polyhedral group. Then $\mathbb{Z} G$ satisfies cancellation and stably-free cancellation if and only if $G$ is one of the seven groups

$$
Q_{8}, Q_{12}, Q_{16}, Q_{20}, T_{24}, O_{48}, I_{120}
$$

(2) ([27, Theorem 15.5 , p. 128]) Cancellation holds for direct products $G=C_{m} \times L$, provided $L$ has cancellation and $C_{m}$ has odd order. By choosing $L$ to be one of the seven groups in (1), and an odd $m$ relatively prime to the order of $L$, we can manufacture an infinite number of groups in class (IV) whose integral group rings satisfy stably-free cancellation.

(3) When $G=Q_{2^{n}}, n \geq 3$, ([27, Theorem 17.7 , p. 138]) implies that the Swan projectives $\langle N, r\rangle$ are either free or not even stably-free. If $p$ is an odd prime, then all Swan projectives for $G=Q_{4 p}$ are free $([27$, Theorem 17.8, p. 138]). Thus these groups satisfy weak cancellation. 
Units in $K_{1}(\mathbb{Z} G)$

Magurn, Oliver, and Vaserstein [16] have developed techniques for determining whether $K_{1}(\mathbb{Z} G)$ is represented by units. They provide the following examples for binary polyhedral groups (class I).

Examples 3.1 (i) $K_{1}\left(\mathbb{Z} Q_{2^{n}}\right)$ is represented by units ([16, Theorem 7.15]).

(ii) For any odd prime $p, K_{1}\left(\mathbb{Z} Q_{4 p}\right)$ is represented by units if and only if the class number $h_{p}$ of the pth cyclotomic field is odd ([16, Theorem 7.16]). Recall that in our notation $Q_{4 p}$ has order $4 p$.

(iii) If $G$ is $T_{24}, O_{48}$, or $I_{120}$, then $K_{1}(\mathbb{Z} G)$ is represented by units ([16, Corollary 7.17]).

(iv) For any prime $p \equiv-1 \bmod 8, K_{1}\left(\mathbb{Z} Q_{16 p}\right)$ is not represented by units $([16$, Theorem $7.18])$.

(v) $S K_{1}(Z G)$ vanishes for a finite abelian group $G$ if and only if either $G \cong\left(C_{2}\right)^{n}$ or each Sylow $p$-subgroup of $G$ has the form $C_{p}$ or $C_{p} \times C_{p^{n}}([16$, p. 325]).

Let $G$ be a group of Type II, III or IV. We now exhibit three different techniques for showing that $K_{1}(\mathbb{Z} G)$ can be represented by units. For many of these groups, this can be reduced to the same question for an appropriate binary polyhedral quotient group. We will need some definitions and results which we state here for convenience.

Let $G$ be a finite group. The group algebra $\mathbb{Q} G$ is semisimple, and hence has a decomposition as a direct sum of simple algebras by Wedderburn's theorem. A component algebra $H$ is simple if and only if its centre is a field $K$. Since $G$ is finite, $K$ is a finite-dimensional extension of $\mathbb{Q}$; therefore, an isomorphic copy of $K$ may be obtained from $\mathbb{Q}$ by adjoining a finite number of roots of irreducible polynomials over $\mathbb{Q}$. A choice of such roots defines an embedding $\rho: K \hookrightarrow \mathbb{C}$

We now single out a type of simple component that is known to cause technical difficulties. For a detailed motivation of the Eichler condition see [4]. The following will suffice for our purposes.

Definition 3.2. A simple $H$ component of $\mathbb{Q} G$ is a non-Eichler component if it is a totally definite quaternion algebra; that is, both of the following conditions hold:

(1) Every embedding of the centre $K$ into $\mathbb{C}$ has image contained in $\mathbb{R}$.

(2) Given an embedding $\rho: K \hookrightarrow \mathbb{R}$, we may always define an extension to $H \hookrightarrow \mathbb{R} \otimes_{K} H$ by identifying elements of $K$ with their image under $\rho$. For every such embedding, we have $\mathbb{R} \otimes_{K} H \cong \mathbb{H}$, where $\mathbb{H}$ is the skewfield of real quaternions.

If either (1) or (2) do not hold, $H$ is called an Eichler component.

Theorem 3.3. ([16, Theorem 6.2, p. 339]). Let $A=V \oplus D$ be a decomposition of finite-dimensional semisimple $\mathbb{Q}$-algebras, and suppose every simple component of $V$ is non-commutative and Eichler. Let $\mathfrak{A}$ be a $\mathbb{Z}$-order in $A$ with image $\mathfrak{D}$ under projection to $D$. Then an element $x$ in $K_{1}(\mathfrak{U})$ is represented by a unit of $\mathfrak{U}$ if and only if its image in $K_{1}(\mathcal{D})$ is represented by a unit of $\mathfrak{D}$. 
Lemma 3.4. Let $M$ be a normal subgroup of the finite group $G$ in the intersection of all normal subgroups $L$ whose associated factor group $G / L$ is binary polyhedral. Let $\mathfrak{H}_{G}$ be the image of $\mathbb{Z G}$ in the sum of non-Eichler components of $\mathbb{Q} G$, and $\mathfrak{W}_{G / M}$ be the image of $\mathbb{Z}(G / M)$ in the sum of non-Eichler components of $\mathbb{Q}(G / M)$. Then $\mathfrak{S}_{G}$ is isomorphic to $\mathfrak{S}_{G / M}$.

Proof. Let $H$ be a simple non-Eichler component of $\mathbb{Q} G$. Let the field $K$ be the centre of $H$, and let $\rho: K \hookrightarrow \mathbb{R}$ be an embedding. Then

$$
H \hookrightarrow \mathbb{R} \otimes_{\mathrm{K}} H \cong \mathbb{H},
$$

where $\mathbb{H I}$ is the skewfield of real quaternions. Let $L$ be the kernel of the composite map

$$
G \hookrightarrow \mathbb{Q} G \hookrightarrow H \hookrightarrow \mathbb{H}
$$

then $G / L$ is isomorphic to a subgroup of $\mathbb{H}^{*}$, whose elements span $\mathbb{H}$ over $\mathbb{R}$. This quotient is nonabelian and hence must be one of the binary polyhedral groups, noncyclic in particular ([4, II p. 305]). We may also identify $G / L$ with a subgroup of $\mathbb{H}^{*}$ whose elements span $\mathbb{H}$ over $\mathbb{Q}$. This implies that $H$ is a non-Eichler component of $\mathbb{Q}(G / L)$. If $M \subset L$ is a normal subgroup of $G$, then $H$ is a simple non-Eichler component of $\mathbb{Q}(G / M)$ as well.

Let $\oplus H_{i}$ be the sum of the simple non-Eichler components of $\mathbb{Q} G$. For each simple $H_{i}$, let $L_{i}$ be the subgroup described above. Consider the surjection $\mathbb{Q} G \rightarrow \mathbb{Q}(G / M)$ induced by $G \rightarrow G / M$. Since $M \subseteq \bigcap L_{i}$ by hypothesis, we have

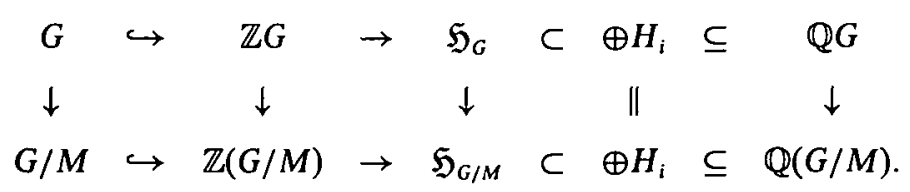

Therefore, the map $\mathbb{Q} G \rightarrow \mathbb{Q}(G / M)$ restricts to an isomorphism $\mathfrak{S}_{G} \cong \mathfrak{H}_{G / M}$.

Theorem 3.5. Let $G$ be a finite group with quotient $Q=G / M$ such that $G^{a b} \rightarrow Q^{a b}$ or $Q \rightarrow Q^{a b}$ splits. Assume also that the simple non-Eichler components of $\mathbb{Q} G$ are among the simple components of $\mathbb{Q} Q$. If $K_{1}(\mathbb{Z} Q)$ and $K_{1}\left(\mathbb{Z} G^{a b}\right)$ are represented by units, then $K_{1}(\mathbb{Z} G)$ is represented by units.

Proof. This proof will be an application of Theorem 3.3. Consider the Milnor square associated to $\mathbb{Z} G$ and the ideals $I_{1}=\operatorname{ker}\left\{\mathbb{Z} G \rightarrow \mathbb{Z} G^{a b}\right\}$ and $I_{2}=\operatorname{ker}\{\mathbb{Z} G \rightarrow \mathbb{Z} Q\}$. Using $\mathbb{Z} G / I_{1} \cong \mathbb{Z} G^{a b}$ and $\mathbb{Z} G / I_{2} \cong \mathbb{Z} Q$ and setting $\mathcal{D}=\mathbb{Z} G /\left(I_{1} \cap I_{2}\right), \bar{\Lambda}=\mathbb{Z} G /\left(I_{1}+I_{2}\right)$, we obtain the commutative square 


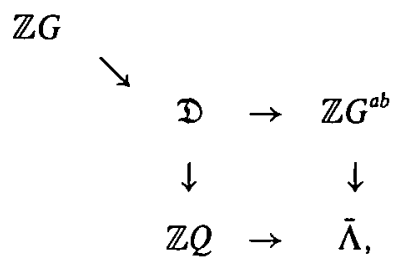

where all maps are surjective. Upon tensoring with $\mathbb{Q}$, we obtain a commutative square of $\mathbb{Q} G$-modules

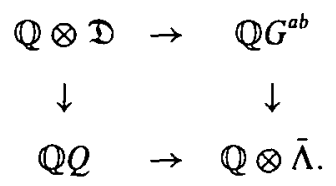

Since $\mathbb{Q} G$ is semisimple and this is a pullback square, the simple components of $D=Q \otimes \mathfrak{D}$ consist of the union of the simple components of $\mathbb{Q} Q$ and $\mathbb{Q} G^{a b}$. By assumption $\mathbb{Q} Q$ contains all of the non-Eichler components of $\mathbb{Q} G$. Therefore $D$ contains all of the commutative and non-Eichler components of $\mathbb{Q} G$. Since $\mathbb{D}$ embeds in $D$, the image of $\mathbb{Z} G$ in $D$ is $D$.

Once we verify that $K_{1}(\mathcal{D})$ is represented by units, all of the assumptions of Theorem 3.3. will be met with $A=\mathbb{Q} G$ and $\mathfrak{U}=\mathbb{Z} G$. We will then be able to conclude that all elements of $K_{1}(\mathbb{Z} G)$ are represented by units. Consider the Mayer-Vietoris sequence associated to the square (3.6):

$$
\begin{gathered}
K_{2}(\mathfrak{D}) \rightarrow K_{2}(\mathbb{Z} Q) \oplus K_{2}\left(\mathbb{Z} G^{a b}\right) \stackrel{\nabla_{2}}{\longrightarrow} K_{2}(\bar{\Lambda}) \\
\stackrel{\mathrm{g}}{\longrightarrow} K_{1}(\mathfrak{D}) \stackrel{\Delta_{1}}{\longrightarrow} K_{1}(\mathbb{Z} Q) \oplus K_{1}\left(\mathbb{Z} G^{a b}\right) \stackrel{\nabla_{1}}{\longrightarrow} K_{1}(\bar{\Lambda}) \ldots
\end{gathered}
$$

The assumed splitting of groups gives rise to similar splittings at the $K_{1}$ and $K_{2}$ levels. Thus $\nabla_{1}$ and $\nabla_{2}$ are surjective, $\partial=0$ and $\Delta_{1}$ is injective. Therefore the image of diagram (3.6) under the functor $K_{1}$ is again a pullback square (of abelian groups).

Any element $x \in K_{1}(\mathcal{D})$ is represented by a pair $\left(x_{1}, x_{2}\right)$ in $K_{1}(\mathbb{Z} Q) \oplus K_{1}\left(\mathbb{Z} G^{a b}\right)$ with $\bar{x}_{1}=\bar{x}_{2} \in \bar{\Lambda}$. According to (3.1) (i) and (v), there are units $\mu_{1}$ in $(\mathbb{Z} Q)^{*}$ and $\mu_{2}$ in $\left(\mathbb{Z} G^{a b}\right)^{*}$ representing $x_{1}$ and $x_{2}$, respectively. Noting that $\bar{\Lambda}$ is commutative and that det $: G L_{\infty}(\bar{\Lambda}) \rightarrow \bar{\Lambda}^{*}$ factors over $K_{1}(\bar{\Lambda})$, we conclude $\bar{\mu}_{1}=\bar{\mu}_{2}$ in $\bar{\Lambda}$. As (3.6) is a pullback, there exists a unit $\mu$ in $\mathfrak{D}$ with projections $\mu_{1}$ and $\mu_{2}$. Since $[\mu] \in K_{1}(\mathcal{D})$ and $x$ have the same projections in $K_{1}(\mathbb{Z} Q)$ and $K_{1}\left(\mathbb{Z} G^{a b}\right)$, respectively, $x$ and $[\mu]$ agree.

Theorem 3.7. Let $G$ be a Type IV group; i.e., of the form $Q \times C_{m}$ with $(m,|Q|)=1$ and $Q$ of Type I, II, or III. If $K_{1}(\mathbb{Z} Q)$ is represented by units, then $K_{1}(\mathbb{Z} G)$ is represented by units. 
Proof. All groups $Q$ of Type I, II, or III abelianize to cyclic groups, $C_{2} \times C_{2}$ or the trivial group. Consequently $G^{a b}$ has cyclic Sylow subgroups or Sylow subgroups of the form $C_{2} \times C_{2}$. Thus $K_{1}\left(\mathbb{Z} G^{a b}\right)$ is generated by units by $(3.1)(v)$. The obvious splitting of $G \rightarrow Q$ carries over to the abelianizations. Now we can apply (3.5).

Definition 3.8. For any $\mathbb{Z}$-order $\mathfrak{A}$ and any rational prime $p$, let $\mathfrak{A}_{p}=\mathbb{Z}_{p} \otimes_{\mathbf{Z}} \mathfrak{U}$ denote the $p$-adic completion of $\mathfrak{U}$. Let

$$
C l_{1}(\mathfrak{U})=\operatorname{ker}\left\{S K_{1}(\mathfrak{U}) \rightarrow \bigoplus_{p} S K_{1}\left(\hat{\mathfrak{U}}_{p}\right)\right\}
$$

where the map is induced by completions at every rational prime.

Theorem 3.9. ([16, Theorem 7.1, p. 342]). Let $A$ be a finite-dimensional semisimple $\mathbb{Q}$-algebra and let $H$ denote the sum of its non-Eichler components. Let $\mathfrak{A}$ be a $\mathbb{Z}$-order in $A$ with image $\mathfrak{H}$ under the projection $A \rightarrow H$. An element $x$ of $K_{1}(A) / C l_{1}(\mathfrak{A})$ is represented by a unit if and only if its image in $K_{1}(\mathfrak{S}) / C l_{1}(\mathfrak{S})$ is represented by a unit of $H$.

To apply these reduction theorems, we either need to know which Eichler components are noncommutative, or be able to compute $C l_{1}$. The following summary of results shows that we may ignore $C l_{1}(\mathfrak{a})$ in many, but not all, cases.

Examples 3.10. (i) For $n \geq 1, C l_{1}\left(\mathbb{Z} Q_{4_{n}}\right)=0$ ([21, Theorem 4]). But for any odd $m$, $C l_{1}\left(\mathbb{Z} Q_{2^{n}} \times C_{m}\right) \cong(\mathbb{Z} / 2 \mathbb{Z})^{\tau(m)-1}$, where $\tau(m)$ is the number of divisors of $m$, including 1 and $m([20$, Theorem 4]).

(ii) If every Sylow $p$-subgroup of $G$ is cyclic, then $C l_{1}(\mathbb{Z} G)=0$. In particular, $\mathrm{Cl}_{1}(\mathbb{Z} G)$ vanishes when $G$ is any group in class II; or when $G=H \times C_{m}$, where $H$ is in class II and $C_{m}$ is a cyclic group with order relative prime to $|G|([20$, Theorem 2]).

(iii) If $G=T\left(8,3^{k}\right)$ is in class III, $C l_{1}(G)=(\mathbb{Z} / 2 \mathbb{Z})^{k-1}([22$, Example 14.4, p. 333]).

Theorem 3.11. If $K_{1}\left(\mathbb{Z} Q_{4(2 l+1)}\right)$ is represented by units, then $K_{1}\left(\mathbb{Z} D\left(2^{k}, 2 l+1\right)\right)$ is represented by units for all $k \geqslant 3$. If $2 l+1$ is prime and the class number $h_{2 l+1}$ is odd, then $K_{1}\left(\mathbb{Z} D\left(2^{k}, 2 l+1\right)\right)$ is represented by units for all $k \geqslant 3$.

Proof. The centre of $G=D\left(2^{k}, 2 l+1\right)$ is a cyclic group of order $2^{k-1}$ generated by $x^{2}$. Since every binary polyhedral group has centre isomorphic to $\mathbb{Z}_{2}$, any homomorphism of $D\left(2^{k}, 2 l+1\right)$ onto a binary polyhedral group must have $\left\langle x^{4}\right\rangle$ in the kernel. Then $M=\left\langle x^{4}\right\rangle$ satisfies the hypotheses of Lemma 3.4, and $D\left(2^{k}, 2 l+1\right) / M \cong Q_{4(2 l+1)}=Q$. Let $q: G \rightarrow Q$ be the projection. By Lemma 3.4 , the image $\mathfrak{S}_{G}$ of $\mathbb{Z} G$ in the sum of the non-Eichler components of $\mathbb{Q} G$ is isomorphic to the image $\mathfrak{S}_{Q}$ of $\mathbb{Z} Q$ in the sum of the non-Eichler components $\mathbb{Q} Q$. The homomorphism $q$ induces a commutative diagram 


$$
\begin{array}{ccc}
K_{1}(\mathbb{Z} G) & \rightarrow & K_{1}\left(\mathfrak{H}_{G}\right) / C l_{1}\left(\mathfrak{H}_{G}\right) \\
\downarrow K_{1}(\mathbb{Z} q) & \downarrow q_{*} \\
K_{1}(\mathbb{Z} Q) & \rightarrow & K_{1}\left(\mathfrak{H}_{Q}\right) / C l_{1}\left(\mathfrak{S}_{Q}\right) .
\end{array}
$$

By the previous step, $q_{*}$ is an isomorphism.

The last assertion follows from the first, by Example 3.1 (ii). From now on, we assume that $K_{1}(\mathbb{Z} Q)$ is represented by units. Let $x \in K_{1}(\mathbb{Z} G)$ and let $y=K_{1}(\mathbb{Z} q)(x)$. Recall from (i) and (ii) of (3.10) that $C l_{1}(\mathbb{Z Q})=0$ and $C l_{1}(\mathbb{Z} G)=0$. By Theorem (3.9), the image $z$ of $y$ in $K_{1}\left(\mathfrak{S}_{Q}\right) / C l\left(\mathfrak{H}_{Q}\right)$ is represented by a unit. Since the image of $x$ in $K_{1}\left(\mathfrak{S}_{G}\right) / C l_{1}\left(\mathfrak{H}_{G}\right)$ equals $q_{*}^{-1}(z)$, a second application of Theorem 3.9 gives that $x$ is represented by a unit.

Theorem 3.12. ([11, Theorem 3, p. 47]). Let $G$ be a finite group. If $K_{1}(\mathbb{Z} H)$ is represented by units for every hyperelementary subgroup $H \subset G$, then $K_{1}(\mathbb{Z} G)$ is represented by units.

Theorem 3.13. If $G=T\left(8,3^{k}\right)$, or $G=T\left(8,3^{k}\right) \times C_{m}$ with $(m, 6)=1$, then $K_{1}(\mathbb{Z} G)$ is represented by units.

Proof. The hyperelementary subgroups of $G$ are among the following types: cyclic, or $Q_{8}$ or $Q_{8} \times C_{l}$ with $l$ odd. All of these groups have $K_{1}$ represented by units. By (3.1) (i) and (v) and Theorem 3.7, the assertion now follows from (3.12). This proof could also have been done using (3.5).

Theorems 3.7, 3.11 and 3.13 show that for a large number of 3-manifold groups $G$, a $(G, 2)$-complex is homotopy equivalent to a spine of a 3 -manifold if and only if it is simple homotopy equivalent to a spine. The following theorem states a stronger result for a particular class of 3-manifold groups:

Theorem 3.14. Let $G$ be one of

$$
Q_{8}, Q_{12}, Q_{16}, Q_{20}, T_{24}, O_{48}, I_{120}
$$

or the direct product of one of the above groups with a cyclic group of relatively prime order. Then all minimal $(G, 2)$-complexes are simple homotopy equivalent to a spine of a 3-manifold.

Proof. This proof essentially consists of verifying the hypotheses of (1.10) and (2.4). That these groups satisfy stably-free cancellation follows from Cancellation Properties (1) and (2). By Example 3.1 (i), (ii), and (iii) and Theorem 3.7, $K_{1}(\mathbb{Z} G)$ is represented by units for all of the groups of the hypothesis. For $Q_{12}$ and $Q_{20}$ we also invoke $h_{3}=1$ and $h_{5}=1$ from ([9, p. 593]). 


\section{REFERENCES}

1. W. Browning, Finite CW-complexes of cohomological dimension 2 with finite abelian $\pi_{1}$, ETH Zürich (unpublished), May 1979.

2. H. Cartan and S. Eilenberg, Homological Algebra (Princeton University Press, Princeton, 1956).

3. M. CoHEN, A Course in Simple-Homotopy Theory (Graduate Texts in Math. 10, SpringerVerlag, New York, 1973).

4. C. W. CURTIS and I. ReINER, Methods of Representation Theory with Applications to Finite Groups and Orders (2 volumes) (John Wiley \& Sons, New York, 1981 and 1987).

5. M. DYER, Homotopy classification of $(\pi, m)$-complexes, J. Pure Appl. Algebra 7 (1976), 249-282.

6. M. Dyer and A. Sieradski, Trees of homotopy types of two-dimensional complexes, Comment. Math. Helv. 48 (1973), 31-44.

7. M. Gutierrez and M. P. Latiolais, Partial homotopy type of finite two-complexes, Math. Z. 207 (1991), 359-378.

8. I. Hambleton and M. KRECK, Cancellation of lattices and finite two-complexes, J. Reine Angew. Math. 442 (1993), 91-109.

9. H. Hasse, Number Theory (Grundlehren der Math. Wiss. 229, Springer-Verlag, Berlin, 1980).

10. C. Hog-Angeloni, W. Metzler and A. Sieradski (editors), Two-dimensional Homotopy and Combinatorial Group Theory (London Mathematical Soc. Lecture Note Series 197, Cambridge University Press, Cambridge, 1993).

11. S. JAJOdia and B. A. Magurn, Surjective stability of units and simple homotopy type, J. Pure Appl. Algebra 18 (1980), 45-58.

12. M. P. Latiolais, Simple homotopy type of finite 2-complexes with finite abelian fundamental group, Trans. Amer. Math. Soc. 293 (1986), 655-662.

13. M. P. Latiolais, When homology implies homotopy equivalence for 2-complexes, $J$. Pure Appl. Algebra 76 (1991), 155-165.

14. E. Luft and D. SJERve, On regular coverings of 3-manifolds by homology 3-spheres, Pacific J. Math. 152 (1992), 151-163.

15. M. Lustig, Nielsen equivalence and simple homotopy type, Proc. London Math. Soc. 62 (1991), 537-562.

16. B. Magurn, R. Oliver and L. Vaserstein, Units in Whitehead groups of finite groups, J. Algebra 84 (1983), 324-360.

17. W. Metzler, Über den Homotopietyp zweidimensionaler CW-Komplexe und Elementartransformationen bei Darstellungen von Gruppen durch Erzeugende und definierende Relationen, J. Reine Angew. Math. 285 (1976), 7-23.

18. W. METzLER, Die Unterscheidung von Homotopietyp und einfachem Homotopietyp bei zweidimensionalen Komplexen, J. Reine Angew. Math. 403 (1990), 201-219.

19. J. Milnor, Groups which act on $S^{n}$ without fixed points, Amer. J. Math. 79 (1957), 623-631.

20. R. Oliver, $S K_{1}$ for finite group rings, I, Invent. Math. 57 (1980), 183-204. 
21. R. Oliver, $S K_{1}$ for finite group rings, III, in Algebraic $K$-Theory (Evanston 1980, Lecture Notes in Math. 854, Springer-Verlag, Berlin, 1981), 299-337.

22. R. Oliver, Whitehead Groups of Finite Groups (London Mathematical Soc. Lecture Note Series, 132, Cambridge University Press, Cambridge, 1988).

23. F. QuinN, Topological quantum invariants and the Andrews-Curtis conjecture (progress report), Virginia Polytechnic Institute \& State University, Blacksburg 1993 (pre-print).

24. P. Scotr, The geometries of 3-manifolds, Bull. London Math. Soc. 15 (1983), 401-487.

25. J.-P. SERRE, Trees (Springer-Verlag, Berlin, 1980).

26. R. G. Swan, Periodic resolutions for finite groups, Ann. of Math. 72 (1960), 267-291.

27. R. G. Swan, Projective modules over binary polyhedral groups, J. Reine Angew. Math. 342 (1983), 66-172.

28. W. Threlfall and H. Seifert, Topologische Untersuchung der Diskontinuitätsbereiche endlicher Bewegungsgruppen des dreidimensionalen sphärischen Raumes, Math. Ann. 104 (1931), $1-70(1930)$.

29. W. Threlfall and H. Seifert, Topologische Untersuchung der Diskontinuitätsbereiche endlicher Bewegungsgruppen des dreidimensionalen sphärischen Raumes (SchluB), Math. Ann. 107 (1933), 543-586 (1932).

30. J. H. C. WhitehEAd, Simple homotopy type, Amer. J. Math. 72 (1950), 1-57.

Department of Mathematical Sciences

Portland State University

PORTLAND, OR 97207-0751

U.S.A. 